\title{
Modeling the power flow in normal conductor-insulator-superconductor junctions
}

\author{
J. Jochum ${ }^{\mathrm{a})}$ \\ Center for Particle Astrophysics, University of California at Berkeley, 301 Le Conte Hall, Berkeley, \\ California 94720 \\ C. Mears \\ Physics and Space Technology Directorate, Lawrence Livermore National Laboratory, P.O. Box 808, \\ L-418, Livermore, California 94551
}

S. Golwala and B. Sadoulet

Center for Particle Astrophysics, University of California at Berkeley, 301 Le Conte Hall, Berkeley, California 94720

J. P. Castle, M. F. Cunningham, and O. B. Drury

Thin Film Laboratory, Department of Physics and Astrophysics, San Francisco State University, 1600 Holloway, San Francisco, California 94132

M. Frank and S. E. Labov

Physics and Space Technology Directorate, Lawrence Livermore National Laboratory, P.O. Box 808, L-418, Livermore, California 94551

F. P. Lipschultz

Thin Film Laboratory, Department of Physics and Astrophysics, San Francisco State University, 1600 Holloway, San Francisco, California 94132

H. Netel

Physics and Space Technology Directorate, Lawrence Livermore National Laboratory, P.O. Box 808, L-418, Livermore, California 94551

B. Neuhauser

Thin Film Laboratory, Department of Physics and Astrophysics, San Francisco State University, 1600 Holloway, San Francisco, California 94132

(Received 27 October 1997; accepted for publication 26 November 1997)

\begin{abstract}
Normal conductor-insulator-superconductor (NIS) junctions promise to be interesting for x-ray and phonon sensing applications, in particular due to the expected self-cooling of the $N$ electrode by the tunneling current. Such cooling would enable the operation of the active element of the sensor below the cryostat temperature and at a correspondingly higher sensitivity. It would also allow the use of NIS junctions as microcoolers. At present, this cooling has not been realized in large area junctions (suitable for a number of detector applications). In this article, we discuss a detailed modeling of the heat flow in such junctions; we show how the heat flow into the normal electrode by quasiparticle back-tunneling and phonon absorption from quasiparticle pair recombination can overcompensate the cooling power. This provides a microscopic explanation of the self-heating effects we observe in our large area NIS junctions. The model suggests a number of possible solutions. (C) 1998 American Institute of Physics. [S0021-8979(98)00406-X]
\end{abstract}

\section{INTRODUCTION}

Normal conductor-insulator-superconductor (NIS) tunnel junctions hold promise for a variety of particle detection applications. ${ }^{1-3}$ The deposition of energy by absorption or scattering of a particle in the normal metal electrode yields a temperature increase of the normal metal; the tunneling current is sensitive to the normal electrode temperature and hence a pulse in the tunneling current results. In addition, NIS junctions are interesting as microcoolers because of an expected self-cooling effect. It has been noted for some time that, under certain bias conditions, the tunneling current is

\footnotetext{
a)Electronic mail: jochum@physics.berkeley.edu
}

dominated by relatively high energy electrons that are replaced by electrons at the Fermi level, and therefore a net transfer of energy out of the normal electrode is possible. Thus, the normal electrode temperature can be reduced below that of the substrate; this effect has been demonstrated for small junctions. ${ }^{4,5}$ The cooling would increase the sensitivity of a detector junction. It could also be used to refrigerate other devices, i.e., to create microcoolers with no moving parts. Even in the absence of self-cooling, improvement of sensitivity with decreasing temperature should be apparent as the bath temperature is decreased. However, most detector junctions fabricated to date show a saturation of the sensitivity as the operating temperature is lowered. Figure 1 shows the theoretical maximal signal height (maximized as a func- 


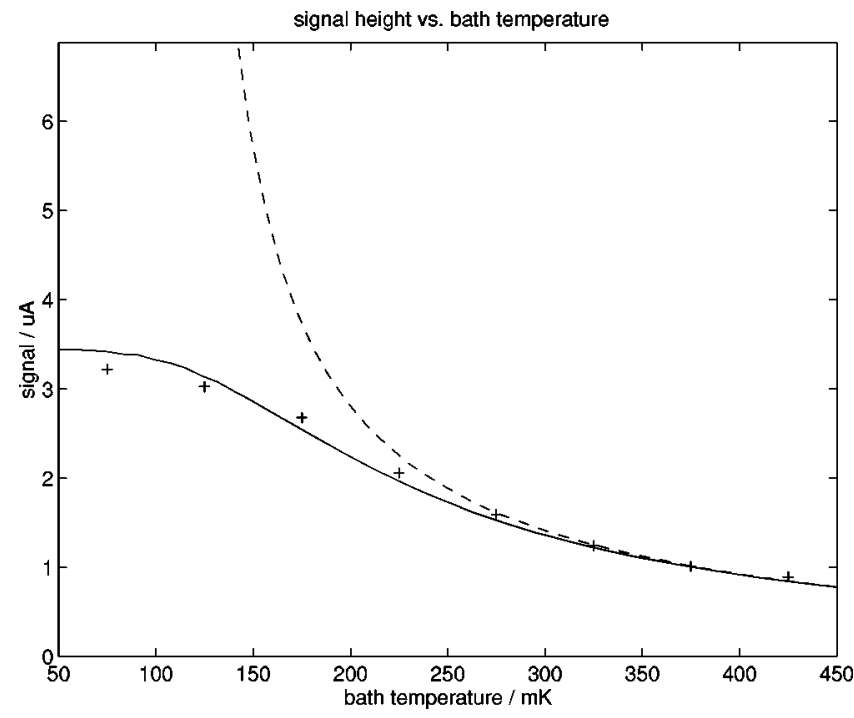

FIG. 1. Maximal signal height (for $5.89 \mathrm{keV} \mathrm{x}$-ray absorption, maximized as a function of bias voltage at fixed $T_{b}$ ) vs bath temperature $T_{b}$. The "optimal" self-cooling model (one in which none of the IV power or the power extracted from the $\mathrm{N}$ electrode is returned to it) is given by the dashed line. In this model, the signal height is expected to strongly increase as the temperature decreases. Such an effect occurs even if the cooling is neglected (i.e., $T_{n}=T_{b}$ is assumed), and the cooling increases the effect by reducing $T_{n}$ below $T_{b}$. In contrast, the measurements (+'s) show a saturation at low $T_{b}$. We believe this apparent heating is due to power returned to the $N$ electrode by back-tunneling currents. A model that includes back-tunneling fits the data well (full line). The method for calculating the expected pulse height with and without these self-heating effects is described in the text.

tion of bias voltage) of a junction with $R_{n}=1 \Omega$ and a tunneling area of $100 \mu \mathrm{m} \times 100 \mu \mathrm{m}$ (dashed line) as a function of bath temperature and compares it to our measurements. (Details of the measurements and the junction fabrication are discussed in Ref. 6.) The observed pulse heights are clearly much lower than expected at low temperature.

A simple explanation for the above behavior is that the entire normal electrode is somehow self-heating above the substrate temperature. An important clue is that the detector junctions typically have a much larger area than those for which cooling has been demonstrated. In such large junctions, electrons that have tunneled from the normal conductor into the superconductor require a much larger time to diffuse out of the tunneling region. Hence, they have a higher probability of engaging in two processes that return energy to the normal electrode. One process is simple quasiparticle back-tunneling. The other is recombination with another quasiparticle, emitting a phonon that may be absorbed in the normal electrode. In this article, we claim that these processes can have a strong effect on the cooling power and so on the device's performance as a detector, and that special care must be taken to remove the tunneled quasiparticles from the tunneling region to ensure self-cooling. To support these points, we present a detailed model of the particle- and power-flows in NIS tunnel junctions, quantitatively describing the effect the back-tunneling current and the recombination phonons have on the cooling power. While the importance of the recombination phonons has been suspected for some time, ${ }^{3}$ we believe we are the first to also consider the effect of backtunneling.
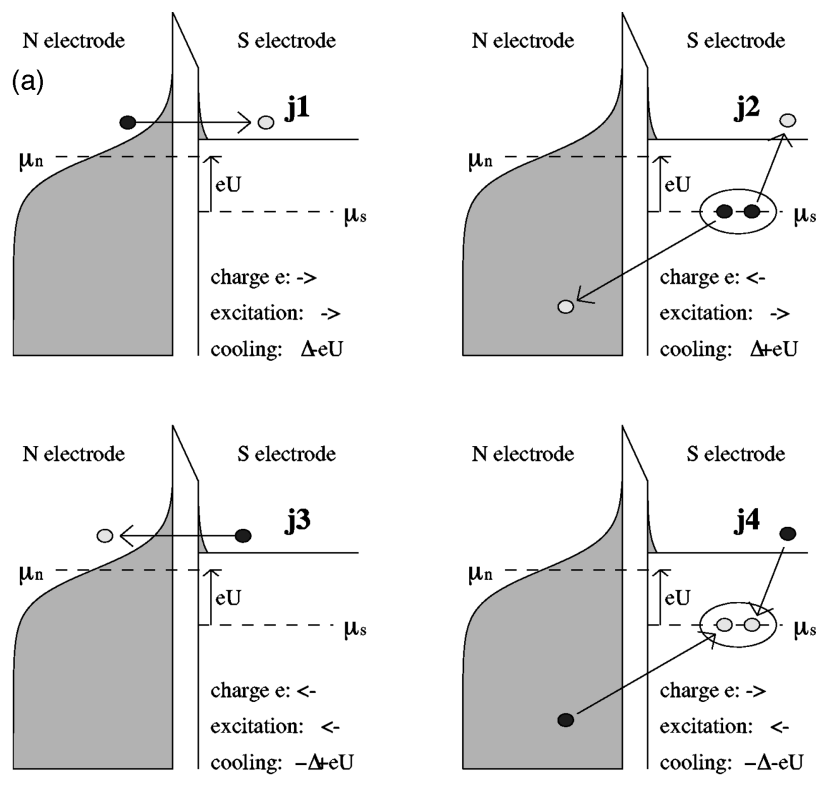

(b)

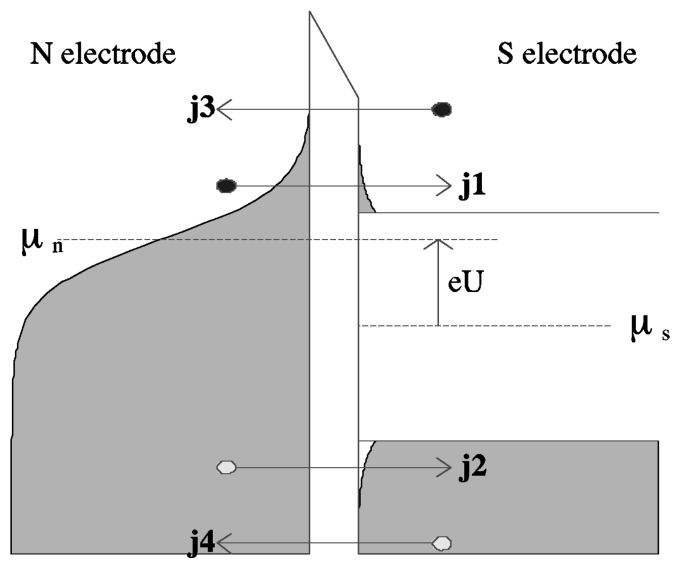

FIG. 2. The four contributions to the NIS tunneling current, displayed in the occupancy diagram. (a) shows the excitation picture and (b) the semiconductor picture. In the latter the direction of the quasiparticle transport is more obvious. The normal conductor is on the left, the superconductor on the right. For two of the currents $\left(j_{1}\right.$ and $\left.j_{3}\right)$, the charge and quasiparticle transport go in the same direction, while for the other two $\left(j_{2}\right.$ and $\left.j_{4}\right)$ the charge and quasiparticle transport are opposite to each other. Hence the current of charges $J_{e}$ and the quasiparticle number current $J_{q}$ differ as discussed in the text. $j_{3}$ and $j_{4}$ are the back-tunneling currents; the energy transferred into the normal conductor is on average much bigger for these currents than the energy removed by the forward tunneling current (composed of $j_{1}$ and $j_{2}$ ).

\section{CURRENTS IN NIS JUNCTION}

In this section, we derive the tunneling and energy currents as determined by the microscopic physics of the system. Please refer to Fig. 2 for a pictorial description of the following.

The tunneling Hamiltonian yielding the transfer of an electrical charge across the tunneling barrier is given $b^{7,8}$

$$
H_{T}=\sum_{s, n, \sigma}\left\{T_{s n} c_{s k \sigma}^{+} c_{n k \sigma}+\text { h.c. }\right\},
$$

where $T_{s n}$ is the tunneling matrix element and $c_{s k \sigma}^{+}$and $c_{n k \sigma}^{+}$ are creation operators for electrons in the superconductor and the normal conductor (with momentum $k$ and spin $\sigma$ ), re- 
spectively. We make the standard assumption that the tunneling process yields no spin flips. If we describe the operators $c_{s k \sigma}^{+}$and $c_{s k \sigma}$ by the modified Bogoliubov-Valatin transformation, ${ }^{7-10}$ using quasielectron creation operators $\gamma_{s k \sigma}^{+}$and operators $S^{+}, S$ that add and remove Cooper pairs to the condensate, then the tunneling terms between eigenstates of the BCS Hamiltonian for the branch $k>k_{F}$ (the momentum at the Fermi energy) are

$$
\begin{aligned}
& T_{s n} c_{s k \sigma}^{+} c_{n k \sigma}+T_{n s} c_{n k \sigma}^{+} c_{s k \sigma}=T_{s n}\left(u_{k}^{*} \gamma_{s k \sigma}^{+}+v_{k} S^{+} \gamma_{s-k-\sigma}\right) c_{n k \sigma}+T_{n s} c_{n k \sigma}^{+}\left(u_{k} \gamma_{s k \sigma}+v_{k}^{*} S \gamma_{s-k-\sigma}^{+}\right) \\
& =\underbrace{T_{s n} u_{k}^{*} \gamma_{s k \sigma}^{+} c_{n k \sigma}}_{j_{1}}+\underbrace{T_{s n} S^{+} v_{k} \gamma_{s-k-\sigma} c_{n k \sigma}}_{j_{4}}+\underbrace{T_{n s} c_{n k \sigma}^{+} u_{k} \gamma_{s k \sigma}}_{j_{3}}+\underbrace{T_{n s} c_{n k \sigma}^{+} S v_{k}^{*} \gamma_{s-k-\sigma}^{+}}_{j_{2}},
\end{aligned}
$$

where $u_{k}$ and $v_{k}$ are the BCS coherence factors. Figure 2(a) provides a schematic description of the four processes. Since in the superconductor destroying an electron and creating a pair is equivalent to destroying a hole and vice versa, $j_{4}$ and $j_{2}$ can be interpreted as hole back- and forward-tunneling, respectively. Figure 2(b) sketches this interpretation in the semiconductor picture: $j_{1}$ and $j_{2}$ are the quasiparticle number currents from the normal conductor into the superconductor, while $j_{3}$ and $j_{4}$ are the corresponding currents in the opposite direction.

There are similar tunneling terms for the branch $k<k_{F}$. If we assume that the two branches $k>k_{F}$ and $k<k_{F}$ are equally populated, we can combine the tunneling terms for them, and the four current contributions are

$$
\begin{aligned}
& j_{1}(\epsilon)=g(\epsilon) f_{n}\left(\epsilon-e U, T_{n}\right)\left(1-f_{s}\left(\epsilon, T_{s}\right)\right) / e^{2} R_{n}, \\
& j_{2}(\epsilon)=g(\epsilon) f_{n}\left(\epsilon+e U, T_{n}\right)\left(1-f_{s}\left(\epsilon, T_{s}\right)\right) / e^{2} R_{n}, \\
& j_{3}(\epsilon)=g(\epsilon)\left(1-f_{n}\left(\epsilon-e U, T_{n}\right)\right) f_{s}\left(\epsilon, T_{s}\right) / e^{2} R_{n}, \\
& j_{4}(\epsilon)=g(\epsilon)\left(1-f_{n}\left(\epsilon+e U, T_{n}\right)\right) f_{s}\left(\epsilon, T_{s}\right) / e^{2} R_{n},
\end{aligned}
$$

where $f_{n, s}(\epsilon)$ are the Fermi functions at $T_{n}$ and $T_{s}$ (the temperatures of the normal conductor and superconductor), respectively. We assume that the electrons in the normal metal and the quasiparticles in the superconductor are each separately in thermal equilibrium. $\epsilon$ is the energy relative to $\mu_{s}$, the Fermi level in the superconductor. In order to express all four currents in terms of $\epsilon>0$, we have used the fact that $1-f_{n}\left(-\epsilon, T_{n}\right)=f_{n}\left(\epsilon, T_{n}\right)$, and $\left|u_{k}\right|^{2}+\left|v_{k}\right|^{2}=1$. The voltage $U$ is the difference of potential between the superconductor and the normal conductor; it is defined to be negative when the Fermi level of the normal electrode is raised above that of the superconductor in the energy diagram given in Fig. 2. The other parameters are: $e$, the electron charge $(<0) ; R_{n}$, the normal resistance of the junction; and $g(\epsilon)=|\epsilon| / \sqrt{\epsilon^{2}-\Delta^{2}}$, the enhancement of the density of states in the superconductor as given by BCS theory.

We will be interested in two combinations of the above currents: $J_{e}$, the number current that gives the electrical current, and $J_{q}$, the net quasiparticle number current. For two of the currents $\left(j_{1}\right.$ and $\left.j_{3}\right)$, the charge and quasiparticle trans- port go in the same direction, while for the other two $\left(j_{2}\right.$ and $j_{4}$ ) the charge and quasiparticle transport are opposite to each other. Hence the current of charges $J_{e}$ and the quasiparticle number current $J_{q}$ differ and are given by

$$
\begin{aligned}
J_{e}=\frac{1}{e} I_{e}= & \int\left(j_{1}-j_{2}-j_{3}+j_{4}\right) d \epsilon \\
= & \frac{1}{e^{2} R_{n}} \int_{\Delta}^{\infty} g(\epsilon)\left(f_{n}\left(\epsilon-e U, T_{n}\right)\right. \\
& \left.-f_{n}\left(\epsilon+e U, T_{n}\right)\right) d \epsilon, \\
J_{q}= & \int\left(j_{1}+j_{2}-j_{3}-j_{4}\right) d \epsilon \\
= & \frac{1}{e^{2} R_{n}} \int_{\Delta}^{\infty} g(\epsilon)\left(f_{n}\left(\epsilon-e U, T_{n}\right)\right. \\
& \left.+f_{n}\left(\epsilon+e U, T_{n}\right)-2 f_{s}\left(\epsilon, T_{s}\right)\right) d \epsilon .
\end{aligned}
$$

The electrical current $I_{e}$ is independent of $T_{s}$ and consequently does not directly depend on the backtunneling currents. On the other hand, the net quasiparticle current does depend directly on the temperature $T_{s}$ of the superconductor and is reduced by a higher $T_{s}$ because of the back-tunneling term $2 f_{s}\left(\epsilon, T_{s}\right)$. If $k T_{n} \ll \Delta+e U$ (a typical biasing condition), then the $f_{n}\left(\epsilon+e U, T_{n}\right)$ term is negligible, and the relation between electrical current and quasiparticle current can be written as

$$
J_{q}=J_{e}-\frac{1}{\tau_{\mathrm{bt}}} N_{s},
$$

where $N_{s} \propto \int 2 g(\epsilon) f_{s}\left(\epsilon, T_{s}\right) d \epsilon$ is the total number of quasiparticles in the $S$ electrode and $\tau_{\mathrm{bt}}$ is the time constant for back-tunneling. If $T_{s}$ is low, $J_{e}$ and $J_{q}$ are equal. In the situation where the superconductor is not in equilibrium, $T_{s}$ should be regarded as a quantity characterizing the number of quasiparticles and Eq. (2.3) should hold.

\section{MICROCOOLER — NIS JUNCTION IN EQUILIBRIUM}

We now calculate the power flow due to the four currents $j_{1}$ through $j_{4}$ and use this result to determine the equilibrium temperatures $T_{n}$ and $T_{s}$. While we noted above that $J_{e}$ does not directly depend on $T_{s}$, we will see that conser- 
vation of power relates $T_{n}$ to $T_{s}$ and thus $J_{e}$ to $T_{s}$ indirectly. We also determine the condition for the normal electrode to cool, i.e., for $T_{n}<T_{b}$.

A simple model for the NIS microcooler holds if the temperatures $T_{n}$ and $T_{s}$ are low compared to $T_{c}$ (the superconductor's critical temperature). In this case, $J_{e}$ is mainly carried by those electrons in the normal electrode with $\epsilon>\Delta-e U$ (i.e., $j_{1}$ ). The removal of these "hot electrons" results in a cooling of the $N$ electrode. However, the assumption of low $T_{s}$ is not always justified, especially in large area junctions where a large number of tunneled quasiparticle can build up in the superconductor, and hence the simple cooling effect is modified.

To investigate this in detail, let us calculate the power carried by the various currents. The net quasihole current $j_{4}-j_{2}$ carries an energy of the order of $\Delta+e U$ into the normal electrode. This is much larger than $\Delta-e U$, the approximate energy carried away by the net quasielectron current $j_{1}-j_{3}$. Note that, because of this asymmetry, even a small back-tunneling current can have a large effect on the cooling power. The power removed from the normal electrode by quasiparticle tunneling is given by

$$
\begin{aligned}
P_{c, e l}= & \int_{\Delta}^{\infty}\left\{(\epsilon-e U)\left(j_{1}-j_{3}\right)-(\epsilon+e U)\left(j_{4}-j_{2}\right)\right\} d \epsilon \\
= & \frac{1}{e^{2} R_{n}} \int\left\{\epsilon\left(j_{1}+j_{2}-j_{3}-j_{4}\right)\right. \\
& \left.-e U\left(j_{1}-j_{2}-j_{3}+j_{4}\right)\right\} d \epsilon \\
\approx & \bar{\epsilon} J_{q}-e U J_{e}=\left(\bar{\epsilon}_{n}-e U\right) J_{e}-\frac{\bar{\epsilon}_{s}}{\tau_{\mathrm{bt}}} N_{s} .
\end{aligned}
$$

$\bar{\epsilon}$ is the mean energy transported by the quasiparticles into the $\mathrm{S}$ electrode. It is an average of $\bar{\epsilon}_{n}$ and $\bar{\epsilon}_{s}$ and is of the order of $\Delta$, with a slight dependence on the bias voltage $U$ $\left(\bar{\epsilon}_{n} \approx \Delta+\frac{1}{2} k T_{n} \quad\right.$ if $\quad k T_{n} \ll \Delta \pm e U$ and $\quad \bar{\epsilon}_{s} \approx \Delta+\frac{1}{2} k T_{s} \quad$ if $\left.k T_{s} \ll \Delta\right)$. The last term on the right hand side of Eq. (3.1) corresponds to back-tunneling and, in the previous literature, has not been included. ${ }^{2,4,5}$ It introduces a dependence of $P_{c, e l}$ on $N_{s}$ and thus on $T_{s} . \tau_{\mathrm{bt}}$ is the average time for backtunneling.

Let us try to understand the origin of the last term, which results in power return to the normal electrode. As noted above, the quasiparticles in $j_{1}-j_{3}$ carry $\Delta-e U$, while the ones in $j_{4}-j_{2}$ carry $\Delta+e U$. Thus, if a quasiparticle tunnels into the superconductor via $j_{1}$ and then tunnels back via $j_{4}$, a net charge $2 e$ has been removed from and a net energy $2 e U$ added to the normal electrode. Integrated over all energies, this is just the IV power provided by the battery, this power must be dissipated somewhere. Cooling of the normal electrode is therefore only possible if the back-tunneling processes are prevented and the tunneled quasiparticles forced to relax (and thus dissipate the IV power) elsewhere. Note that this Joule heating should not be confused with heating of the normal metal due to its intrinsic resistance.

Another important process that participates in dissipating the IV power is the generation of $2 \Delta$ phonons by quasipar- ticle self-recombination in the superconductor: this provides another mechanism for quasiparticles to relax. However, some of these phonons can reach and be absorbed in the normal metal, thus yielding another power flow into the $\mathrm{N}$ electrode. Their contribution is equivalent to the backtunneling of two quasiparticles at the gap energy. We take this into account by adding an extra term to the power flow, yielding

$$
\begin{aligned}
P_{c, \text { tot }} & =P_{c, e l}-2 \Delta \kappa \frac{R N_{s}^{2}}{2} \\
& \approx\left(\bar{\epsilon}_{n}-e U\right) J_{e}-\frac{\bar{\epsilon}_{s}}{\tau_{b t}} N_{s}-\Delta \kappa R N_{s}^{2},
\end{aligned}
$$

where $R N_{s}^{2}$ is the quasiparticle self-recombination rate. Since two quasiparticles are required to create one phonon of energy $2 \Delta$, the phonon emission rate is $\left(R N_{s}^{2}\right) / 2 . \kappa$ is the fraction of recombination phonons absorbed in the $N$ electrode (the remainder are assumed to be lost to the bath).

It must also be noted that there may be other paths for quasiparticle relaxation for which the energy released is somehow removed. Examples would be out-diffusion of quasiparticles from the junction area (so that when they do recombine, the probability of the $2 \Delta$ phonons reaching the $N$ electrode or the junction area is negligible) or trapping of the quasiparticles in a normal metal or lower gap superconductor trap (with the assumption that the relaxation phonons emitted during trapping also cannot reach the junction region or the normal electrode). We characterize these processes by a simple time constant $\tau_{\text {out }}$.

We determine $T_{s}$ by requiring zero net quasiparticle flow into the superconductor; this will then determine the above power flows and thereby help to fix $T_{n}$. We assume that the number of thermally-generated quasiparticles at $T_{b}$ is negligible, and thus $N_{s}$ is determined only by the previously discussed processes. The number conservation equation is then

$$
0=J_{q}-\frac{1}{\tau_{\text {out }}} N_{s}-R N_{s}^{2}=J_{e}-\frac{1}{\tau_{\text {so }}} N_{s}-R N_{s}^{2}
$$

with $\tau_{\text {so }}^{-1}=\tau_{\text {bt }}^{-1}+\tau_{\text {out }}^{-1}$.

As noted above, $N_{s} / \tau_{\text {out }}$ describes the disappearance of quasiparticles by means other than self-recombination, such as out-diffusion or more active methods such as trapping. $\tau_{\text {so }}$ is the time a quasiparticle remains in the junction area of the superconducting electrode and is determined by the time constant for out-diffusion/trapping $\left(\propto \tau_{\text {out }}^{-1}\right)$ and for backtunneling $\left(\propto \tau_{\mathrm{bt}}^{-1}\right)$. For low currents, i.e., $J_{e} \ll 1 / 4 R \tau_{\text {so }}^{2}$, we find

$$
N_{s} \approx \tau_{\mathrm{so}} J_{e}
$$

and from Eq. (3.2)

$$
P_{c, \mathrm{tot}} \approx\left\{\Delta\left(1-\frac{\tau_{\mathrm{so}}}{\tau_{\mathrm{bt}}}\right)-e U\right\} J_{e} .
$$

The cooling term $\Delta J_{e}$ of $P_{c, e l}$ is reduced by a factor $\left(1-\tau_{\mathrm{so}} / \tau_{\mathrm{bt}}\right)$. If the quasiparticles are not removed effectively from the tunneling area, i.e., $\tau_{\text {out }} \rightarrow \infty$ and $\tau_{\text {so }} \rightarrow \tau_{\text {bt }}$, the 


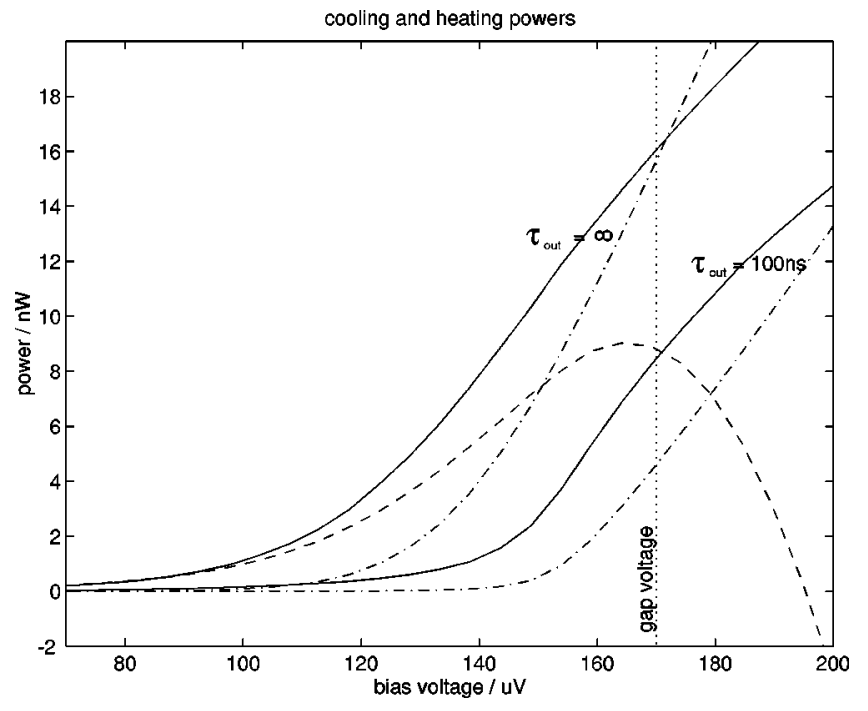

FIG. 3. Power flow into the normal electrode via back-tunneling quasiparticles (full line) and recombination phonons (dash-dotted line) for a junction with $R_{n}=0.1 \Omega$ and size as given in the text. The two sets of lines show these heating powers for average out-diffusion times of $\tau_{\text {out }}=\infty$ and $100 \mathrm{~ns}$. The phonon reabsorption was taken to be $\kappa=0.2$ in each case. As $\tau_{\text {out }}$ decreases, so does the back-tunneling power. For comparison, the dashed line shows the expected cooling power $P_{c, 0}$ in the absence of back-tunneling and recombination phonon absorption. If $P_{c, 0}$ is smaller than the sum of the two heating powers, the normal electrode is heated.

cooling effect is largely reduced and one is left with the IV power $e U J_{e}$. The condition for cooling $\left(P_{c, \text { tot }}>0\right)$ is

$$
\frac{\Delta-e U}{\Delta}>\frac{\tau_{\mathrm{so}}}{\tau_{\mathrm{bt}}}
$$

For example, if the junction is biased at $e U=0.9 \Delta$, the time $\tau_{\mathrm{so}}$ required for a quasiparticle to leave the tunneling area in the superconductor must be more than ten times faster than the time for back-tunneling $\tau_{\mathrm{bt}}$ in order to preserve the cooling effect! In Eqs. (3.5) and (3.6) it was assumed that the power returning from the superconductor to the normal conductor is dominated by the back-tunneling term $\bar{\epsilon}_{s} N_{s} / \tau_{\mathrm{bt}}$. A limit similar to Eq. (3.6), replacing $\tau_{\mathrm{so}} / \tau_{\mathrm{bt}}$ by $\kappa$, can be derived for the case the recombination phonons carry most of the returning power. Figure 3 shows the returning powers carried by back-tunneling quasiparticles and by recombination phonons for situations typical in our measurements. It gives the result of the integral in equation Eq. (3.1) for a $\mathrm{Al} / \mathrm{AlOx} / \mathrm{Cu}$ junction with $R_{n}=0.1 \Omega$. We assume $\kappa=0.2$, which, as we will show, best fits our data. $\kappa$ depends mainly on the relative sizes of the phonon absorption length and the normal electrode film thickness (2000 $\AA$ typically). $\tau_{\text {out }} \approx 100 \mathrm{~ns}$ is a typical out-diffusion time for a very good film of thickness of $2000 \AA$ for a junction area of $100 \mu \mathrm{m}$ $\times 100 \mu \mathrm{m}$. The back-tunneling time constant $\tau_{\mathrm{bt}}$ for such a junction is about $1 \mu \mathrm{s}$. For the calculation we have assumed a gap of $170 \mu \mathrm{eV}$ and a quasiparticle self-recombination rate constant in the aluminum ${ }^{11}$ of $52 \mu \mathrm{m}^{3} \mathrm{~s}^{-1} . R$ is given by this constant divided by the volume of the film.

We have now determined $N_{s}$ and therefore $T_{s}$ in terms of $T_{n}$ (via $J_{e}$ ). To determine $T_{n}$, we require zero net power flow into the $N$ electrode. In addition to the power carried by tunneling quasiparticles and recombination phonons, we must also include the power $P_{\text {ep }}$ exchanged with the thermal phonon bath via the electron-phonon coupling in the normal electrode. (At the temperatures of interest, the thermal impedances between the phonons of the junction, those of the substrate, and those of the bath are much less than the impedance between the electrons and phonons of the normal electrode, and so we assume the thermal phonons are effectively the bath.) With $\Sigma$ being the electron-phonon coupling constant and $V_{n}$ the volume of the normal electrode, the balance of the heat flows into the $N$ electrode is given by

$$
\begin{aligned}
0=P_{c, \text { tot }}+P_{\mathrm{ep}} \approx & \left(\bar{\epsilon}_{n}-e U\right) J_{e}-\frac{\bar{\epsilon}_{s}}{\tau_{\mathrm{bt}}} N_{s}-\Delta \kappa R N_{s}^{2} \\
& -\Sigma V_{n}\left(T_{b}^{5}-T_{n}^{5}\right) .
\end{aligned}
$$

For copper we take ${ }^{4} \Sigma=2 \mathrm{nW} \mathrm{K}^{-5} \mu \mathrm{m}^{-3}$. Together with the characteristics $I_{e}\left(U_{b}, T_{n}\right)$ and the solution $N_{s}\left(I_{e}\right)$ from Eqs. (3.3), (3.7) can be used to determine $T_{n}\left(U_{b}\right)$ by numerical root-finding methods. In the case of cooling ( $P_{c \text {,tot }}$ positive), $T_{n}$ drops below the substrate temperature $T_{b}$.

In summary, we have the following picture of the NIS junction: the Joule power $I_{e} U$ and the heat removed from the $N$ electrode by the tunneling electrons are carried by the quasiparticles into the $S$ electrode. The fraction of this power that is returned to the normal electrode is determined by competition between, on the one hand, back-tunneling and recombination phonon generation, and, on the other hand, quasiparticle removal mechanisms. The back-tunneling power and the recombination phonons can easily overcompensate the cooling power, and it is therefore crucial for microcooler applications that the tunneled quasiparticles be quickly removed from the superconductor, especially from the tunneling region where back-tunneling and phonon transmission are most probable.

\section{DETECTOR — NIS JUNCTION DYNAMIC BEHAVIOR}

In this section, we determine the response of the NIS junction to an energy deposition in the presence of the various effects discussed above. We do this by again requiring conservation of quasiparticle number in the superconductor and conservation of power in the normal metal.

Consider the deposition of an energy $E$ in the $N$ electrode, yielding a rise $\delta T_{n}$ in $T_{n}$. As a result, the currents $J_{e}$ and $J_{q}$ and the number of quasiparticles in the $\mathrm{S}$ electrode $N_{s}$ exhibit time dependent increases $\delta J_{e}, \delta J_{q}$, and $\delta N_{s}$. These variations yield changes in $P_{c \text {,tot }}$ and $P_{\text {ep }}$ that restore the equilibrium temperature $T_{n} . \delta T_{n}(t)$ can be measured via the change $\delta I_{e}(t)$ of the electrical current. We assume that the processes of establishing internal equilibrium in each element of the system (the normal electrode at $T_{n}$, the superconductor at $T_{s}$, and the bath, fixed at $T_{b}$ ) are much faster than the time scales of interest, and hence we can parameterize the behavior entirely in terms of temperatures and thermal distribution functions. 
To determine the behavior of $N_{s}$, we write the nonequilibrium version of Eqs. (2.3) and (3.3) by expanding all quantities around their equilibrium values and canceling the equilibrium terms. This yields

$$
\begin{aligned}
& \delta J_{q}=\delta J_{e}-\frac{1}{\tau_{\mathrm{bt}}} \delta N_{s}, \\
& \delta \dot{N}_{s}=\delta J_{e}-\frac{1}{\tau_{s}} \delta N_{s}
\end{aligned}
$$

with $\tau_{s}^{-1}=\left(2 R N_{s}+\tau_{\text {so }}^{-1}\right)$.

The change in the number of quasiparticles in the superconductor $\delta \dot{N}_{s}$ is given by the changes in the recombination rate $2 R N_{s} \delta N_{s}$, in the out-diffusion and back-tunneling rate $\delta N_{s} / \tau_{\text {so }}$, and in the incoming current $\delta J_{e}$. The time dependent $\delta N_{s}$ follows $\delta J_{e}$ with a delay of $\tau_{s}$. This delay is equal to the effective time a quasiparticle is present in the superconducting electrode.

To determine $\delta T_{n}$, we perform the same procedure on the $N$ electrode power conservation equation [cf., Eq. (3.7)]

$$
\begin{aligned}
C \delta \dot{T}_{n}= & -\left(\frac{d P_{c, \text { tot }}}{d T_{n}}+\frac{d P_{\mathrm{ep}}}{d T_{n}}\right) \delta T_{n}+P_{\text {dep }} \\
= & -\left(\bar{\epsilon}_{n}-e U\right) \delta J_{e}+\left(\frac{\bar{\epsilon}_{s}}{\tau_{\mathrm{bt}}}+2 \Delta \kappa R N_{s}\right) \\
& \times \delta N_{s}-5 \Sigma V_{n} T_{n}^{4} \delta T_{n}+P_{\mathrm{dep}},
\end{aligned}
$$

where $P_{\text {dep }}$ is the initial power deposition [ $=E \delta(t)$ for x-ray absorption or scattering]. The other terms on the right side correspond to the change in the tunneling cooling power and the change in the heat flow from the thermal phonons. The left side is of course the rate of change of the energy of the $N$ electrode. If we assume $\tau_{s} \ll \tau_{d}$ (the time scale of the signal, see below), e.g., the out-diffusion is very fast, then $\delta N_{s}$ can be taken to be proportional to $\delta J_{e}$ and one can solve the above equations to find

$$
\delta T_{n}(t)=\frac{E}{C} e^{-t / \tau_{d}},
$$

where the natural relaxation time of the normal metal temperature is

$$
\tau_{d}=\frac{C}{G_{\mathrm{ep}}+G_{\mathrm{tun}}} .
$$

$C$ is the heat capacity of the normal electrode,

$$
G_{\text {ep }}=\frac{d P_{\text {ep }}}{d T_{n}}=5 \Sigma V_{n} T_{n}^{4}
$$

is the thermal conductance to the substrate through the electron-phonon coupling and

$$
G_{\text {tun }}=\frac{d P_{c, \text { tot }}}{d T_{n}}
$$

is the effective thermal conductance due to tunneling. The electrical signal $\delta I_{e}(t)$ is proportional to $\delta T_{n}(t)$ and is simply given by

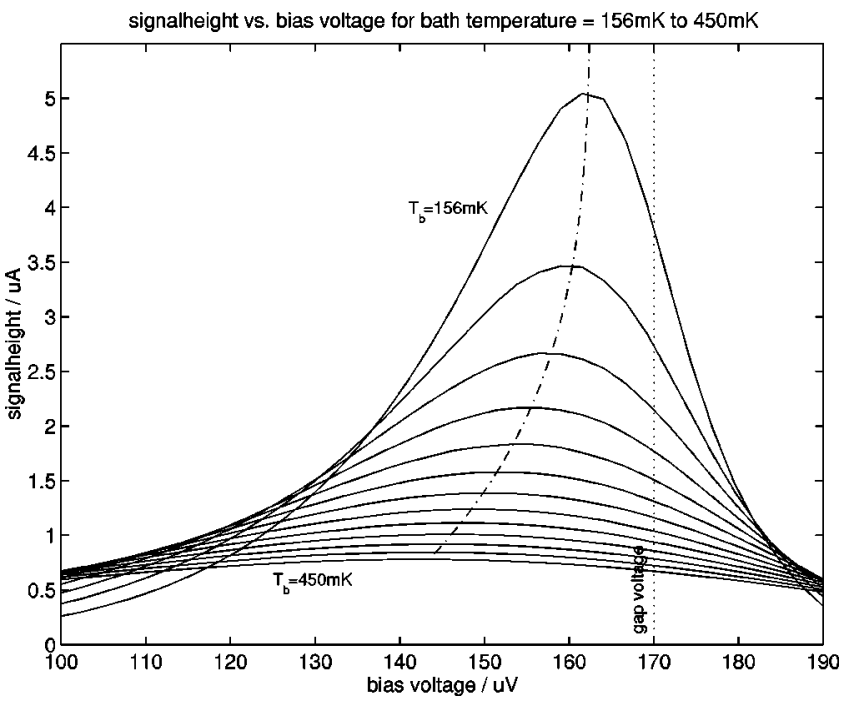

FIG. 4. Pulse height (for $5.89 \mathrm{keV}$ x-ray deposition) of a NIS junction for temperatures $T_{b}$ from $156 \mathrm{mK}$ to $450 \mathrm{mK}$. This pulse height is proportional to $(1 / C)\left(d I_{e} / d T_{n}\right)$. We assume perfect cooling here: no back-tunneling and no recombination phonon heating of the normal electrode. As the temperature decreases, the maximal pulse height increases. We define the optimum bias voltage to be the voltage at which this maximal pulse height occurs (not necessarily the same as the bias that gives the optimal energy resolution); this optimum bias voltage moves closer to the superconductor's gap voltage as the temperature is lowered. The dash-dotted line tracks the optimum bias voltage with temperature.

$$
\delta I_{e}(t)=e \delta T_{n}(t) \frac{d J_{e}}{d T_{n}} .
$$

Note that, with the same approximations regarding outdiffusion and operating temperature and bias, we may relate $G_{\text {tun }}$ to the electrical current and its temperature derivatives:

$$
G_{\mathrm{tun}}\left[\bar{\epsilon}_{n}-e U-\tau_{s}\left(\frac{\bar{\epsilon}_{s}}{\tau_{\mathrm{bt}}}+2 \Delta \kappa R N_{s}\right)\right] \frac{d J_{e}}{d T_{n}}+\frac{1}{2} k e J_{e},
$$

where the last term comes from the temperature dependence of $\bar{\epsilon}_{n}$. This approximation shows how $G_{\text {tun }}$ is reduced by the backtunneling current, (i.e., as $\tau_{s} / \tau_{\mathrm{bt}}$ increases), and by the absorption of recombination phonons, (i.e., as $\kappa$ increases).

In the more general case where $\tau_{s}$ is not small compared to $\tau_{d}$, the signal shape will show two time constants, a rise and a decay time, approximately equal to $\tau_{s}$ and $\tau_{d}$.

\section{CONSEQUENCES FOR MICROCOOLERS AND DETECTORS}

We have demonstrated that back-tunneling quasiparticles and absorption of recombination phonons have significant effects on the power flow in NIS junctions. In this section we discuss the practical impact this has on the performance of NIS junctions as microcooler and particle detectors.

We have seen that $T_{n}$, and not $T_{b}$, primarily determines the performance characteristics of NIS detector junctions, i.e., quantities such as pulse height $I_{0}$ and decay time $\tau_{d}$. An important point is that, as $T_{n}$ is decreased, $(1 / C)\left(d I_{e} / d T_{n}\right)$ continues to increase and hence the pulse height improves; 
clearly, then, utilization of the cooling effect also improves the junction's particle detection characteristics. Figure 4 demonstrates this for the junction described earlier by showing the pulse height as a function of the bias voltage $U$ at different bath temperatures, under the assumption of no back-tunneling or recombination phonon absorption: clearly, the maximal pulse height continues to increase as the temperature decreases. We also point out that the optimum bias voltage (the voltage corresponding to maximal pulse height) is a useful diagnostic of the normal electrode temperature: it should approach the superconductor's gap voltage as $T_{n}$ decreases. However, as discussed in the introduction, the data exhibit quite different behavior that suggests elevation of $T_{n}$ above $T_{b}$.

We also note that the back-tunneling quasiparticles, as well as the absorption of recombination phonons, yield increased thermal and shot noises and thus also contribute to a degradation of the signal-to-noise ratio relative to an ideal case. A detailed discussion of the noise in NIS junctions is beyond the scope of this article and will be reported separately.

Before comparing our model to the data, we first exclude two other possible explanations for the low temperature saturation seen in Fig. 1. One is parasitic Joule dissipation in the normal metal electrode. Under the bias conditions for the above data set, the expected self-cooling power is an order of magnitude larger than Joule dissipation due to the resistance of the normal metal film, and so we expect that such heating plays a negligible effect. Another possibility is smearing of the superconducting electrode's energy gap. If the smearing is much smaller than $\Delta-e U$, as in our case, one can model this as a variation of the temperature over the junction, some regions being colder and others warmer. The colder regions yield a larger pulse height than the nominal value, while the warmer ones yield a smaller pulse height. However, the increase due to the cooler regions is larger than the decrease due to the warmer regions (as one can infer from the dashed line in Fig. 1), and hence we expect any reasonable gap smearing to increase the maximal pulse height from the BCS gap expectation. We believe that self-heating by the backtunneling currents and recombination phonons provide a better explanation of the data.

We apply our model to the $\mathrm{A} / \mathrm{AlOx} / \mathrm{Cu}$ junction described in Sec. III. In the worst case scenario, there is no out-diffusion to aid the removal of quasiparticles in the superconducting electrode from the tunneling area; i.e., $\tau_{\text {out }}$ $\rightarrow \infty$. In Fig. 3, we compare the cooling power $P_{c, 0}$ calculated in the absence of back-tunneling current (i.e., $\tau_{\mathrm{bt}}^{-1}=\kappa=0$ ) with the heat flow back from the superconductor due to back-tunneling quasiparticles, $\bar{\epsilon} N_{s} / \tau_{\mathrm{bt}}$. In the case of no out-diffusion of quasiparticles, the heat input always is larger than the nominal cooling power $P_{c, 0}$, resulting in a net heating power $P_{c \text {,tot }}$ into the normal electrode. The situation improves if out-diffusion increases. In the optimum case, the mean free path for electrons is only limited by the film thickness. For a film thickness of $2000 \AA$, the time needed for a localized electron density to diffusively spread over $100 \mu \mathrm{m}$ is about $100 \mathrm{~ns}$. If diffusion is this fast, the heating power from back-tunneling is reduced, and a re-

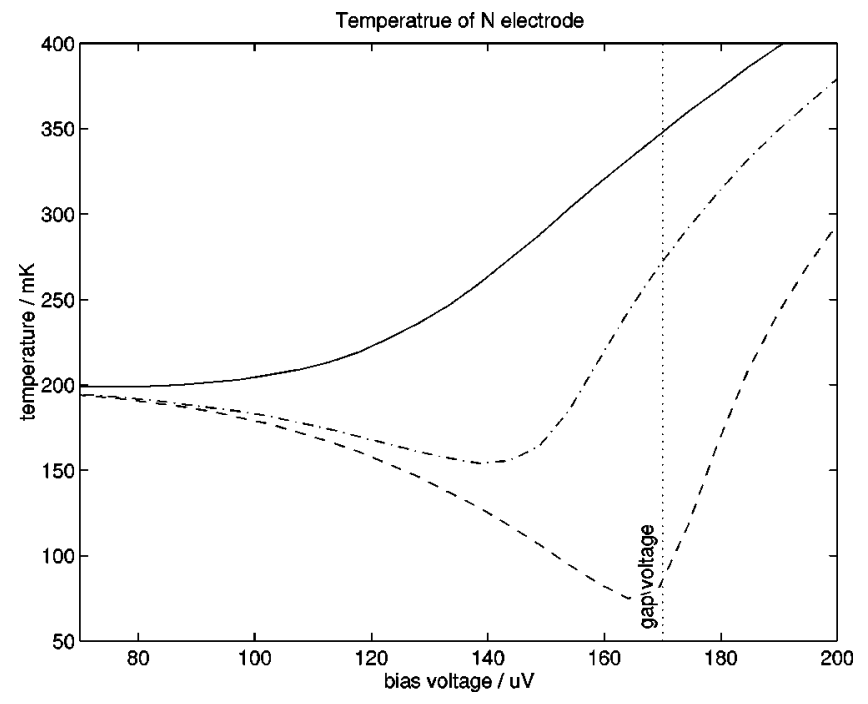

FIG. 5. Temperature $T_{n}$ of the normal electrode vs bias voltage at a bath temperature of $T_{b}=200 \mathrm{mK}$ for a junction with $R_{n}=0.1 \Omega$ and size as given in the text. The full lines show the temperature for $\tau_{\text {out }}=\infty$ and $100 \mathrm{~ns}$ and a phonon reabsorption fraction of $\kappa=0.2$. The dashed line shows the temperature expected from $P_{c, 0}$, ignoring back-tunneling and recombination phonon absorption.

gion of bias voltage for which cooling occurs begins to appear. The upper limit of this region moves closer to the gap; this fact is advantageous in terms of the detection sensitivity, as can be seen from Fig. 4. Figure 5 shows the temperature of the normal electrode versus the bias voltage. Faster outdiffusion clearly aids cooling of the normal electrode.

We have taken pulse height and optimum bias voltage data for a large area junction with the same parameters as our model junction. More details about the fabrication of the junctions and the experimental setup for the data are dis-

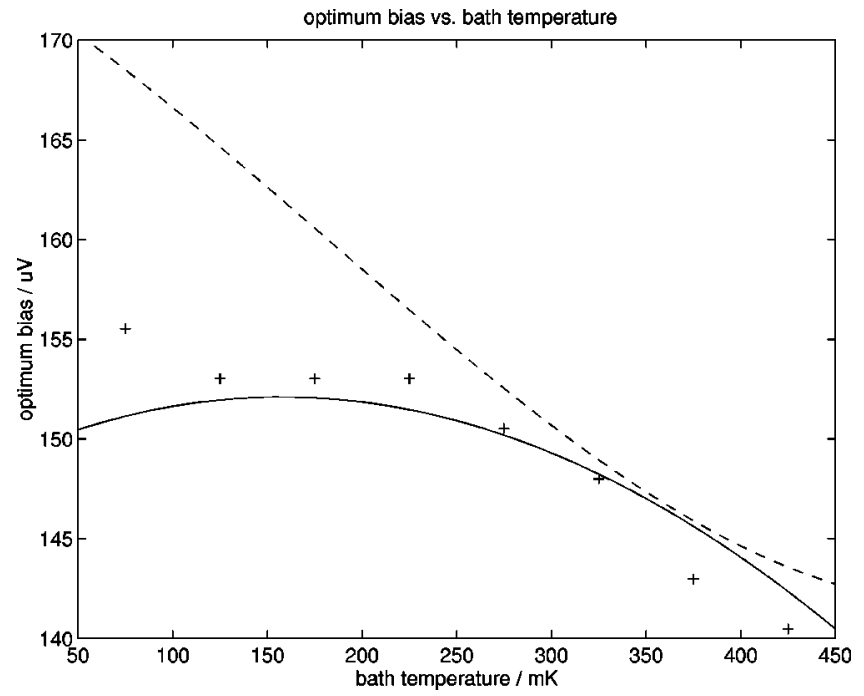

FIG. 6. As the bath temperature is decreased, the optimum bias point should increase towards the gap voltage (dashed line, see also Fig. 4). Our data (+), on the other hand, display a saturation of this quantity, indicating that $T_{n}$ does not decrease beyond some point. The full line gives the optimum bias voltage for a model in which $\tau_{\text {out }}=\infty, \kappa=0.2$, suggesting that backtunneling/recombination phonon effects give a reasonable explanation of the data. 
cussed in Ref. 6. Figure 1 shows the pulse height for 5.89 $\mathrm{keV}$ x-ray absorption from our measurements. As the full line shows, the data are well matched by the model with $\tau_{\text {out }}=\infty$ and $\kappa=0.2$. The optimum bias voltage data (Fig. 6) qualitatively matches the self-heating model also. Having acquired some confidence in the validity of the model, we are experimenting with some practical ideas to ameliorate the behavior of these devices: (a) changing the aspect ratio of the junction window from square to long and narrow in order to reduce the mean distance out of the junction area; (b) increasing the thickness of the superconducting electrode to reduce the back-tunneling rate; and (c) placement of trapping layers (either of normal conductors or superconductors of smaller gap) near the junction in order to trap tunneled electrons and thus reduced back-tunneling. These ideas all serve to address the need to make $\tau_{\text {bt }}$ large while reducing $\tau_{\text {out }}$. (It is also important to maintain a low recombination phonon absorption rate, but the solutions of the two problems are the same.) For example, the aspect ratio change exploits the fact that the average time needed for out-diffusion strongly depends on the dimensions of the junction. With the same film quality, but dimensions of only $10 \mu \mathrm{m} \times 10 \mu \mathrm{m}$, the average out-diffusion time is a factor of 100 faster than for the case of the $100 \mu \mathrm{m} \times 100 \mu \mathrm{m}$ junction. The out-diffusion time could also be reduced by improvement of the diffusion constant of the superconducting film, but this is more difficult to achieve practically. Another example is the way in which the back-tunneling time can be increased by increasing the superconducting electrode's volume, $V_{s}$, by thickening the film underneath the junction. In effect, this simply dilutes the quasiparticle density near the junction by providing a greater volume for quasiparticle diffusion away from the junction. One might also consider increasing the resistance to increase the back-tunneling time. But this must be balanced against the fact that the nominal cooling power $P_{c, 0}$ is itself inversely proportional to $R_{n}$.

\section{CONCLUSIONS}

We have demonstrated that back-tunneling of quasiparticles and absorption of recombination phonons can have a strong negative impact on the performance of large area NIS junctions that are typically employed for detector applications. The rate at which quasiparticles back-tunnel depends on the time they remain in the superconducting electrode. As the model presented here shows, this time has to be at least an order of magnitude shorter than the time constant for back-tunneling in order for cooling to occur and the expected detector performance to be achieved. With this time as a parameter fitted to the pulse height and optimum bias voltage data, we will be able to evaluate the success of different measures taken to reduce the back-tunneling currents. Strategies for doing so include designing junctions with extreme aspect ratios, increasing the thickness of the superconducting electrode, and attaching quasiparticle traps to the superconducting electrode.

\section{ACKNOWLEDGMENTS}

This work was supported in part by the Center for Particle Astrophysics, an NSF Science and Technology Center, under Cooperative Agreement AST-912005, and by IGPPLLNL Grant No. AP96-41. J. Jochum is partly supported by a Feodor-Lynen Fellowship of the Alexander von Humboldt Foundation.

${ }^{1}$ A. Benoit, M. Martin, and B. Pannetier, J. Low Temp. Phys. 93, 727 (1993).

${ }^{2}$ M. Nahum, J. M. Martinis, and S. Castles, J. Low Temp. Phys. 93, 733 (1993).

${ }^{3}$ M. Nahum and J. M. Martinis, Appl. Phys. Lett. 66, 3203 (1995).

${ }^{4}$ M. Nahum, T. M. Eiles, and J. M. Martinis, Appl. Phys. Lett. 65, 3123 (1994).

${ }^{5}$ M. M. Leivo, J. P. Pekola, and D. V. Averin, Appl. Phys. Lett. 68, 1996 (1996).

${ }^{6}$ O. B. Drury, J. P. Castle, S. R. Golwala, J. Jochum, B. Neuhauser, C. A. Mears, W. C. Fellers, F. P. Lipschultz, and B. Sadoulet, Proceedings of the 7th International Workshop on Low Temperature Detectors (LTD-7), July 27-Aug. 2, 1997, Munich, Germany, edited by S. Cooper, (Max Planck Institute of Physics, Munich, Germany, 1997), Vol. A26, p. 56.

${ }^{7}$ J. Bardeen, Phys. Rev. Lett. 9, 147 (1962).

${ }^{8}$ M. Tinkham, Introduction to Superconductivity (McGraw-Hill, New York, 1975)

${ }^{9}$ N. N. Bogoliubov, Nuovo Cimento 7, 794 (1958).

${ }^{10}$ J. Valatin, Nuovo Cimento 7, 843 (1958).

${ }^{11}$ J. N. Ullom, P. A. Fisher, and M. Nahum, Proceedings of the 7th International Workshop on Low Temperature Detectors LTD-7, July 27-Aug 2, 1997, Munich, Germany, edited by S. Cooper, (Max Planck Institute of Physics, Munich, Germany, 1997), Vol. D4, p. 142. 